\title{
Dorsal and Ventral Parietal Contributions to Spatial Orienting in the Human Brain
}

\author{
Ana B. Chica, ${ }^{1}$ Paolo Bartolomeo, ${ }^{1,2,3 *}$ and Antoni Valero-Cabré ${ }^{4,5,6 *}$ \\ ${ }^{1}$ INSERM-U975, Centre de Recherche de l'Institut du Cerveau et de la Moëlle Epinière (CRICM) and Université Pierre et Marie Curie (UPMC), Groupe \\ Hospitalier Pitié-Salpêtrière, F-75013 Paris, France, ${ }^{2}$ Assistance Publique-Hôpitaux de Paris, Groupe Hospitalier Pitié-Salpêtrière, Fédération de \\ Neurologie, 75013 Paris, France, ${ }^{3}$ Department of Psychology, Catholic University, 20123 Milan, Italy, ${ }^{4}$ Centre National de la Recherche Scientifique (CNRS) \\ UMR 7225, Centre de Recherche de l'Institut de Cerveau et da la Moëlle Epinière (CRICM), Université Pierre et Marie Curie (UPMC), 75651 Paris, France, \\ ${ }^{5}$ Laboratory for Cerebral Dynamics, Plasticity, and Rehabilitation, Department of Anatomy and Neurobiology, Boston University School of Medicine, \\ Boston, Massachusetts 02118, and ${ }^{6}$ Cognitive Neuroscience and Information Technology Research Program, School of Psychology, Open University of \\ Catalonia (UOC), 08018 Barcelona, Spain
}

Influential functional magnetic resonance imaging (fMRI)-based models have involved a dorsal frontoparietal network in the orienting of both endogenous and exogenous attention, and a ventral system in attentional reorienting to task-relevant events. Nonetheless, given the low temporal resolution and susceptibility to epiphenomenal activations of fMRI, such depictions remain highly debated. We hereby benefited from the high temporal resolution and causal power of event-related transcranial magnetic stimulation to explore the implications of key dorsal and ventral parietal regions in those two types of attention. We provide for the first time causal evidence of right intraparietal sulcus involvement in both types of attentional orienting, while we link the temporoparietal junction with the orienting of exogenous but not endogenous spatial attention.

\section{Introduction}

Spatial orienting can be driven either endogenously, by weighting learned predictive contingencies between top-down cues and events; or exogenously, by nonpredictive bottom-up signals, reflexively orienting our systems to a given region of the visual space. Behavioral paradigms typically require participants to respond to targets preceded by peripheral visual cues, so that when most targets appear at the validly cued location, participants are usually faster to respond to valid than invalid targets. However, when cues are spatially uninformative about the future location of relevant targets, they induce a short lasting facilitation followed by a performance cost at the valid location, known as inhibition of return (IOR) (Berlucchi, 2006; Klein, 2000).

Although the endogenous/exogenous taxonomy has been substantiated at the behavioral level (Klein, 2004), the existence of perfectly segregated brain networks in charge of each of those types remains elusive. Relevant neuropsychological evidence from right parietal damage patients found such lesions associated to impairments in contralesional exogenous orienting, with a relative sparing of endogenous orienting (Bartolomeo and Chok-

\footnotetext{
Received 0ct. 19, 2010; accepted Nov. 17, 2010.

This work was supported by postdoctoral grants from the Neuropôle de Recherche Francilen (NeRF) and Marie Curie Intra-European Program (FP7) to A.B.C., and FP6 and Agence National de la Recherche Scientifique (ANR) project eraNET-NEURON “BEYONDVIS" (to A.V.-C.). We are thankful to Bastien Oliviero and Romain Valabregue for their help in data analysis.

*P.B. and A.V.-C. share senior authorship.

Correspondence should be addressed to either Ana B. Chica or Antoni Valero-Cabré, CRICM Hôpital Salpêtrière, 47 Boulevard de I'Hôpital, F-75013 Paris, France. E-mail: anachica@ugr.es or avalerocabre@gmail.com.

DOI:10.1523/JNEUROSCI.5463-10.2010

Copyright $\odot 2011$ the authors $\quad 0270-6474 / 11 / 318143-07 \$ 15.00 / 0$
}

ron, 2002), thus strongly suggesting the anatomical segregation of those two systems. In contrast, in patients with damage on the temporoparietal junction (TPJ), Friedrich et al. (1998) reported extinction-like patterns (related to exogenous orienting) for contralesional targets with larger effects in TPJ than in parietal patients, hence emphasizing the role of parietal regions in the attentional disengagement of endogenous orienting (see also $\mathrm{He}$ et al., 2007).

On the basis of functional magnetic resonance imaging (fMRI) data, Corbetta and Shulman (2002) put forward a model in which spatial orienting depends on the activity of a bilateral frontoparietal network, whereas a ventral frontoparietal network would be responsible for responding to unexpected task-relevant targets, and would thus be a plausible candidate to underlie exogenous orienting. Surprisingly, a study by Kincade et al. (2005) found no modulation on the ventral network during the attentional orienting period, favoring the notion of a single attentional orienting system (implicated in both endogenous and exogenous orienting), supported by the dorsal frontoparietal network. The ventral network would be exclusively implicated in reorienting to behaviorally relevant targets (Hahn et al., 2006; Corbetta et al., 2008).

In this study, we used transcranial magnetic stimulation (TMS) to noninvasively interfere with time-specific neural events at circumscribed brain areas and provide causal evidence about the potential implication of key dorsal and ventral parietal sites on the computations leading to endogenously or exogenously driven attentional orienting. We delivered time locked pairs of TMS pulses at key intervals for attentional processing induced by endogenous or exogenous cues during the execution of a visual 
discrimination task. In separate blocks, the right intraparietal sulcus (IPS) and TPJ were targeted, whereas sham TMS was used as a control. To measure the impact of such interventions, we used a variant of the Posner cue-target task (Posner, 1980).

In the exogenous task, a spatially noninformative peripheral cue was presented at either 200 or 800 ms before target onset. Facilitation was to be expected at the short stimulus onset asynchrony (SOA), while inhibition (IOR) should follow at the longer SOA (Posner and Cohen, 1984). In the endogenous task, the exact same peripheral cue was used, but it predicted (67\% of the times) the appearance of the target at the same location of the cue. In this case, a facilitation of visual discrimination was expected mostly at the short SOA, while no inhibition was to be induced due to the allocation of endogenous attention at the cued location (Chica et al., 2006; Chica and Lupiáñez, 2009). We hypothesized that TMS would interfere with the contribution of cortical areas crucial for cue-induced endogenous or exogenous attentional orienting and that such effects would prove region (TPJ or IPS) and attention modality (endogenous vs exogenous) specific.

\section{Materials and Methods}

Participants. Sixteen participants (8 women, one left-handed; mean age of 28 years, $S D=5$ ) gave their signed informed consent to participate in the study. Participants had no neurological or psychiatric conditions and followed all the safety requirements to undergo MRI and TMS interventions. They reported to have normal or corrected to normal vision and audition and were all naive to TMS stimulation. The study was reviewed by the INSERM ethical committee and received the approval of an Institutional Review Board (CPP Ile de France 1). Participants underwent first a structural MRI, which was reconstructed three-dimensionally (3D) and served to navigate the position the TMS coil. They then carried over two independent sessions separated by at least $2 \mathrm{~d}$ (endogenous and exogenous). Each session consisted of three runs of an attentional paradigm under three conditions: real TMS on two right hemisphere cortical areas (right IPS and TPJ) and sham TMS. The order of the two sessions and the three TMS conditions within each session was counterbalanced across participants.

Apparatus and stimuli. A Windows-based computer running a stimulus presentation software (meyeParadigm, www.eye-brain.com) was used to control the presentation of visual stimuli and behavioral data collection. Another computer running specialized software (ClearView, Tobii Technology AB), was used to monitor correct fixation (temporal and spatial resolution: $50 \mathrm{~Hz} ; 0.25^{\circ}$ ). Stimuli were presented on the eye tracker screen (Tobii Technology AB, 17 inches wide, $1024 \times 768,16 \mathrm{~ms}$ refresh rate). The fixation point consisted of a white “+" $\operatorname{sign}\left(1 \times 1^{\circ}\right)$ presented at the center of the display in a black background. Two white placeholder squared boxes $\left(5^{\circ}\right.$ height $\times 3^{\circ}$ width) were displayed, with their center situated at $7^{\circ}$ to the left and right of the fixation cross. The to-be-discriminated target consisted of a white " $\mathrm{X}$ " or "O" letter $\left(1 \times 1^{\circ}\right)$, and was presented at the center of one of the two placeholders.

Procedure. Every trial started with a fixation display. After $500 \mathrm{~ms}$, the peripheral cue was presented with equal probability within the left or right marker for $50 \mathrm{~ms}$. Another $50 \mathrm{~ms}$ after cue offset, two singly triggered TMS pulses, separated by $25 \mathrm{~ms}$, were delivered within a time window of $50 \mathrm{~ms}$. The target was presented either 50 or $650 \mathrm{~ms}$ later, with an SOA of 200 and $800 \mathrm{~ms}$, respectively, with regard to cue onset. On half of the trials the target was the character "X" and on the other half it was an "O". This was presented for $33 \mathrm{~ms}$, and was equally likely to appear inside the left or right placeholder. Participants were required to discriminate target's identity by pressing one of two adjacent keys with the index and central finger of their right hand (ipsilateral to the TMS stimulated hemisphere) with a maximal response window of $2000 \mathrm{~ms}$ from target onset. Response mapping was counterbalanced between participants. The use of the right hand prevented response times (RTs) from being contaminated by direct or indirect TMS activations of the left motor cortex. Furthermore, no hand or arm muscle activation was detected during the experiment. In case no key press was detected during the response win- dow or in the event of an incorrect response, visual feedback was provided in the computer screen for $500 \mathrm{~ms}$. During the intertrial interval the screen remained black. Its duration was adjusted trial to trial so that it was never inferior to $5 \mathrm{~s}$.

Participants performed two evaluation sessions (endogenous and exogenous), separated by at least $2 \mathrm{~d}$, and completed them in a counterbalanced order across participants. In the exogenous task, the peripheral cue was spatially not informative about the future location of the target, and thus was presented at the same location than the target on half of the trials and at the opposite location on the other half (50\% valid trials, $50 \%$ invalid trials). In the endogenous task, the cue was spatially informative about the future location of the target and it was presented at the same location as the target on $67 \%$ of the trials, and at the opposite location on $33 \%$ of the trials (67\% valid trials, $33 \%$ invalid trials). The order of the sessions was counterbalanced between participants.

In the nonpredictive cue session, each block consisted of 192 trials, while in the predictive cue session each block consisted of 288 trials. In each session participants performed 3 blocks of trials in which we either stimulated the right IPS or the right TPJ, or we carried over a sham run with the TMS coil edge against the right parietal bone in an area between IPS and TPJ, positioned at $90^{\circ}$ with the coil' surface away from the skull. This procedure was effective in TMS naive participants mimicking the TMS associated sensations, while preventing the magnetic field from significantly reaching and stimulating the brain. The order of the 3 TMS conditions was counterbalanced between participants. The same response keys and block order were kept in each participant for both evaluation sessions.

TMS targeting, neuronavigation, and pulse delivery. Structural T1weighted MRI scans were acquired for all the participants at the CENIR MRI center (Salpêtrière Hospital of Paris). We used a 3T Siemens MPRAGE (magnetization-prepared rapid-acquisition gradient echo), flip-angle $=9$, repetition time $=2300 \mathrm{~ms}$, echo time $=4.18 \mathrm{~ms}$, slice thickness $=1 \mathrm{~mm}$. Right TPJ and IPS coordinates were chosen from a previous event-related fMRI study, which determined the task activated brain areas involved in the endogenous and exogenous orienting of spatial attention (Kincade et al., 2005). In this study, the right IPS ( $x, 16 ; y$, $-63 ; z, 47$; Fig. 1) was activated during the cue period when participants oriented attention endogenously. In this same study, right TPJ $(x, 51 ; y$, $-51 ; z, 26$; Fig. 1) failed to show any modulation during the cue-target interval, but displayed its activation peak during the target phase, when presented at unexpected locations.

SPM5 software (UCL, London, UK) running under Matlab 7.4 license (MathWorks) was used to localize and mark these two regions in each individual brain. We first created the regions of interests in the MNI space using the Marsbar toolbox for Matlab (http://marsbar.sourceforge. net/). The structural images of the participants were segmented into white and gray matter. The regions created using the coordinates from the study by Kincade et al. (2005) were de-normalized using the inverse segmentation matrix created for each participant (spatial smooth isotropic Gaussian kernel of $1 \mathrm{~mm}$ full-width half-maximum). The resulting regions were coregistered with the participant's structural image, which resulted in the precise location of this area for each individual brain.

TMS procedure and navigation. The two TMS pulses were delivered during $50 \mathrm{~ms}$. The first pulse started $50 \mathrm{~ms}$ after cue offset, and either 50 or $650 \mathrm{~ms}$ before target onset by means of a biphasic repetitive stimulator (Superapid2, The MagStim Company) and a set of $70 \mathrm{~mm}$ TMS figure of eight coil which was held tangentially to the skull with the axis of the coil angled $\sim 45^{\circ}$ from the mid-sagittal axis (lateral to medial and caudal to rostral). The TMS coil was positioned on the two areas of interest by means of a $2 \mathrm{~mm}$ precision neuronavigation system (eXimia NBS System, Nexstim) with the capacity to estimate and track in real time the relative position of our figure of eight coil on the sectional and 3D reconstruction of the participant's MRI. The two areas of interest, right IPS and TPJ, were localized in the MRI 3D reconstruction and labeled so that the coil and the perpendicular projection of the estimated magnetic field accurately coincided with the foci of such areas. All participants received stimulation at suprathreshold levels with respect to their individual motor thresholds. We attempted the use of a fixed TMS intensity of $80 \%$ maximum stimulator output throughout all the participants, cortical 

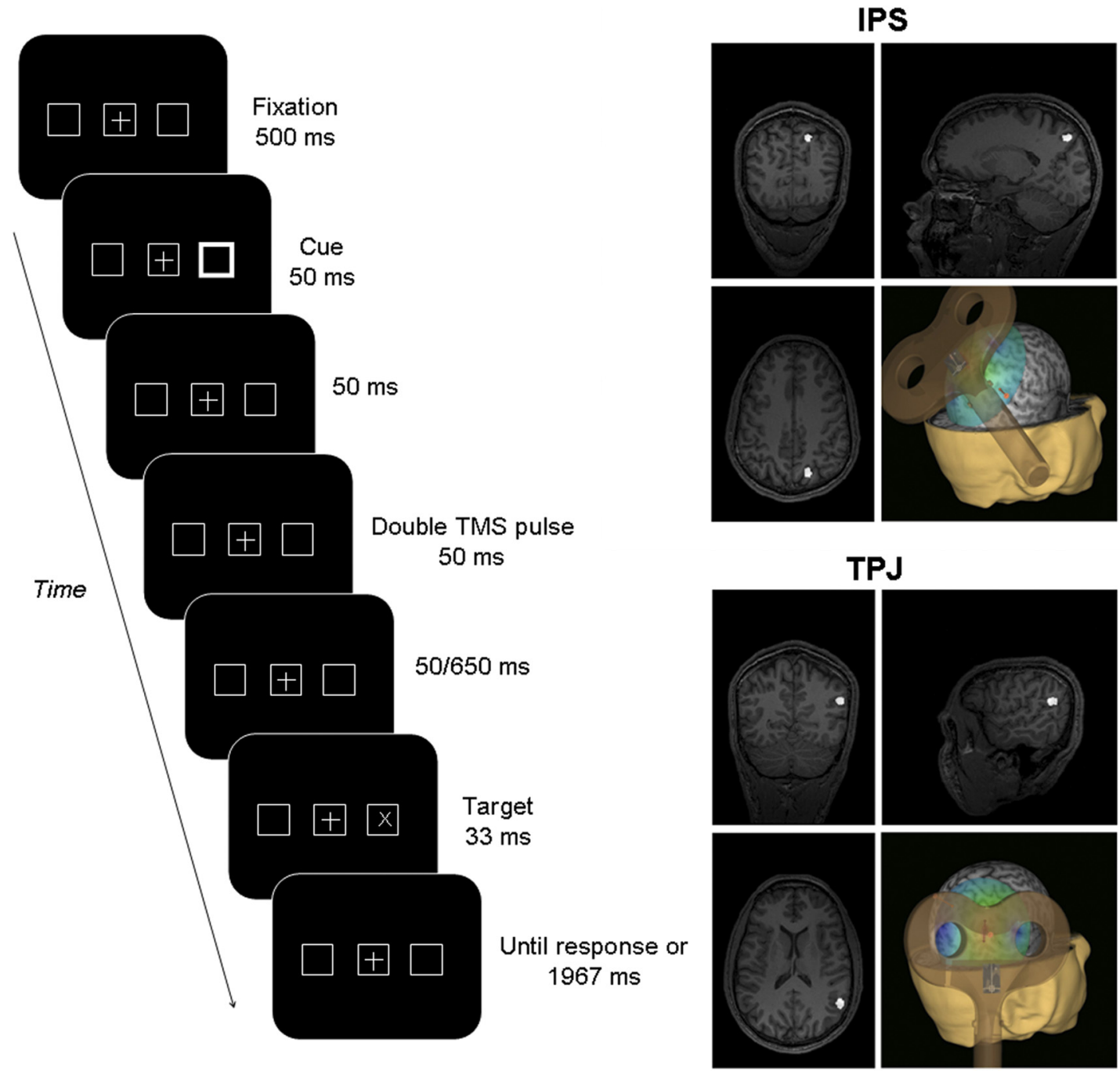

Figure 1. Sequence and timing of events in a given trial. The localization of IPS and TPJ in one of our participants is also shown.

sites, and experimental conditions. Nonetheless, stimulation was adapted in those cases in which TMS induced facial or tongue sensations, involuntary blinks, or jaw activations until those events were no longer induced.

Behavioral data analysis. Three participants had to be excluded from the analyses because their RTs were abnormally slow $(>2.5 \mathrm{SD}$ from the participants' mean data in at least one of the conditions). For the remaining 13 participants, RTs above or below $2.5 \mathrm{SD}$ were removed from the analysis, which accounted for $2.66 \%$ of trials in the exogenous task, and $2.48 \%$ in the endogenous task.

Technical problems to record eye movements were encountered in 18 of the 78 sessions performed by the 13 participants included in the final analysis. These problems were due to bad quality calibrations in participants with light-colored eyes, glasses, or truncated and incomplete recordings due to eye-tracker software failures. In the sessions with usable data, participants broke fixation in only $5 \%$ of the trials for the exogenous task, and in $7 \%$ for the endogenous task.

In our experiment, both accuracy and RT were emphasized. It is well known that under these conditions, speed-accuracy trade-off-based strategies can be adopted. For example, participants can respond faster but less accurately to attended targets. We thus used inverse efficiency (IE, RT/proportion correct), a measure that combines RT and accuracy (Townsend and Ashby, 1983). IE provides an index of behavioral effects, which is free of speed-accuracy trade-offs.

For both endogenous and exogenous tasks, mean IE scores were submitted to a repeated-measures ANOVA, with the intraparticipant factors of side (cues presented in the right-ipsilateral hemifield, or left-contralateral hemifield, to the TMS stimulation site), TMS condition (sham, right IPS, right TPJ interventions), validity (valid, invalid trials), and cue to target SOA (200, $800 \mathrm{~ms})$.

\section{Results}

Exogenous attentional orienting task

Validity effects largely differed for cues presented in the ipsilateral and contralateral visual hemifields (Fig. 2; Table 1). This was reflected by a significant interaction between cue side, TMS condition and validity, $F_{(2,24)}=4.57, \mathrm{MSE}=607, p=0.021$. Previously planned ANOVAs were performed for ipsilateral and contralateral cues. Ipsilaterally, there was a significant interaction between validity and SOA, $F_{(2,24)}=10.55, \mathrm{MSE}=1109, p=$ 0.007 , that did not depend on the TMS condition (interaction between validity, SOA, and TMS condition, $F<1$ ). Figure 2 shows the expected modulation of the validity effect for ipsilateral cues in the sham TMS condition, consisting of facilitation at the short SOA, and inhibition at the longer SOA. This same pattern of facilitation for short and inhibition for long SOAs remained unaltered during the two active TMS conditions.

In contrast, for contralateral cues, i.e., for cues displayed on the left visual hemifield, such cue-validity effects critically depended on the TMS condition, $F_{(2,24)}=4.68, \mathrm{MSE}=795, p=$ 


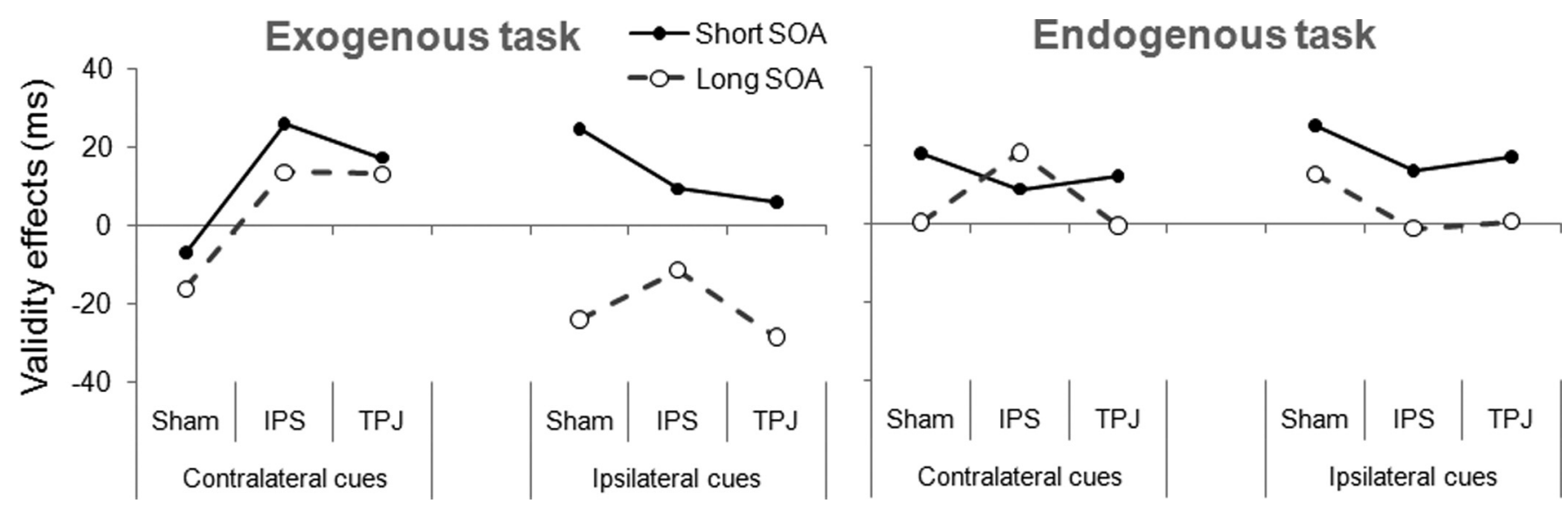

Figure 2. Validity effects for the exogenous and the endogenous tasks. The $y$-axis represents mean validity effects (invalid minus valid), for the inverse efficiency scores (RT/proportion correct). Positive values indicate facilitatory effects (larger IE scores for valid than for invalid trials), while negative values indicate inhibitory effects (larger IE scores for invalid than for valid trials).

0.019. In the sham TMS condition, responses were slower and less accurate for valid than for invalid trials at both the long and also the short SOA. In contrast, when either IPS or TPJ were stimulated, a significant facilitatory effect was observed, $F_{(2,12)}=9.24$, MSE $=877, p=0.010$, at either SOA (interaction between validity and SOA, $F<1)$.

In sum, the TMS interference of either IPS or TPJ prevented the expected contralateral inhibition at the long SOA when attention was reflexively oriented, suggesting the causal implication of both right-lateralized cortical areas in exogenously driven attentional orienting.

\section{Endogenous attention task}

The use of endogenous cues induced a significant main effect of validity on target discrimination, $F_{(1,12)}=7.52$, $\mathrm{MSE}=954, p=$ 0.018 , that interacted with SOA, $F_{(2,24)}=7.31, \mathrm{MSE}=429, p=$ 0.013 . Given the predictive value of the cue, no IOR was observed at the long SOA. The facilitatory effects produced by the cue did not depend on stimulus side or the stimulated region (interaction between side, TMS condition and validity, $F<1$ ). Facilitatory effects were observed at the short SOA regardless of the three TMS conditions for both ipsilateral and contralateral targets. Furthermore, facilitatory effects at the long SOA were exclusively observed after TMS stimulation on IPS for contralateral cues. This paradoxical TMS-induced effect was confirmed by a direct comparison of contralateral IPS with the sham TMS condition. In particular, an ANOVA of contralateral cues including IPS and the sham TMS as conditions demonstrated a marginally significant interaction between TMS condition, validity and SOA, $F_{(2,24)}=4.59, \mathrm{MSE}=257, p=0.053$. Furthermore, post hoc comparisons (Fisher LSD) revealed a significant facilitatory effect for the sham condition at the short but not at the long SOA ( $p=$ 0.014 , and $p=0.925$, respectively), and a significant facilitation at the long but not at the short SOA after IPS stimulation ( $p=$ 0.013 , and $p=0.178$, respectively).

It is worth remarking that analyzing raw RT is a more standard measure than IE. Most of the times, RT and IE yield similar results. However, this is not the case in the present study. For the exogenous task, a similar validity $\times$ region $\times$ side interaction, $F_{(2,24)}=3.19, p=0.059$, to the one reported in the IE measure is observed when analyzing raw RT. For the endogenous task, however, the raw RT analysis shows a region $\times$ validity interaction, $F_{(2,24)}=4.24, p=0.026$, with a facilitatory effect for both sham and IPS stimulation, while no facilitation at all is observed for TPJ stimulation. Therefore, from the raw RT data we could conclude that TPJ plays a role in the endogenous orienting of attention. However, the accuracy data show a marginally significant interaction between region, validity and $\mathrm{SOA}, F_{(2,24)}=2.70, p=0.08$, in the endogenous task. This interaction showed a facilitatory effect in both the sham and TPJ condition for the short SOA. It is not fair to conclude, using the RT, that participants are not orienting attention endogenously when TPJ is stimulated, given that the accuracy measure shows a normal orienting. Therefore, in the present experiments IE represents more accurately our data than raw RT.

In sum, for endogenous attentional orienting, TMS on IPS but not TPJ induced abnormal facilitatory effects at long SOAs. Such results implicate IPS but not TPJ in the processing underlying the orienting of endogenous attention by means of predictive informative cues.

\section{Control experiments}

A collateral finding in our exogenous task was the absence of facilitation at $200 \mathrm{~ms}$ SOA under sham TMS. In consequence, any interpretation of potential stimulation effects for this specific condition and task requires extreme caution. By means of a control experiment, we first tested if such finding could have resulted from the lateralized TMS generated sound, acting as an unexpected cue-back, producing IOR at short SOAs (Prime et al., 2006). A group of participants $(n=16)$ performed a single block of the exogenous task under sham TMS, provided through a coil placed in the midline of the participants parietal scalp. We found a significant validity $\times$ SOA interaction, $F_{(1,15)}=14.64, \mathrm{MSE}=$ 3643, $p=0.001$, that did not depend on cue side (left or right), $F<1$. Facilitatory effects were statistically similar for left and right cues ( 48 and $63 \mathrm{~ms}$, respectively, $F<1$ ), thus suggesting that under those conditions such facilitatory effects were present and robust.

To rule out the potential role of a lateralized TMS generated noise in such absence of facilitation, we tested in a new control experiment $(n=6)$, whether our IPS and TPJ interference results could be replicated when opposing the TMS lateralized noise with that of an additional sham TMS coil discharging in a homotopic left hemisphere location. A repeated-measures ANOVA with experiment (original experiment and control with bilateral stimulation) added as a between participant factor, confirmed the results presented for the exogenous block, demonstrating a significant interaction between cue side, region and validity, 
Table 1. Mean RTs (in milliseconds) and proportion correct (in parentheses) for each experimental condition

\begin{tabular}{|c|c|c|c|c|c|c|c|c|c|c|c|c|}
\hline \multirow[b]{3}{*}{ SOA } & \multicolumn{6}{|c|}{ Contralateral (left) cues } & \multicolumn{6}{|c|}{ Ipsilateral (right) cues } \\
\hline & \multicolumn{2}{|l|}{ Sham } & \multicolumn{2}{|l|}{ IPS } & \multicolumn{2}{|l|}{ TPJ } & \multicolumn{2}{|l|}{ Sham } & \multicolumn{2}{|l|}{ IPS } & \multicolumn{2}{|l|}{ TPJ } \\
\hline & 200 & 800 & 200 & 800 & 200 & 800 & 200 & 800 & 200 & 800 & 200 & 800 \\
\hline \multicolumn{13}{|c|}{ Exogenous cues } \\
\hline Valid & $527(0.96)$ & $523(0.98)$ & $526(0.99)$ & $515(0.99)$ & $524(0.98)$ & $503(0.98)$ & $527(1.00)$ & $505(0.96)$ & $525(0.97)$ & $526(0.99)$ & $523(0.98)$ & $515(0.97)$ \\
\hline Invalid & $531(0.98)$ & $505(0.98)$ & $541(0.97)$ & $519(0.97)$ & $534(0.97)$ & $507(0.96)$ & $541(0.98)$ & $502(1.00)$ & $537(0.98)$ & $512(0.98)$ & $524(0.97)$ & $497(0.99)$ \\
\hline \multicolumn{13}{|c|}{ Endogenous cues } \\
\hline Valid & $525(0.98)$ & $519(0.98)$ & $506(0.97)$ & $493(0.98)$ & $525(0.97)$ & $508(0.98)$ & $526(0.98)$ & $509(0.98)$ & $504(0.97)$ & $496(0.96)$ & $523(0.98)$ & $517(0.97)$ \\
\hline Invalid & $537(0.97)$ & $525(0.99)$ & $513(0.97)$ & $506(0.97)$ & $524(0.95)$ & $508(0.98)$ & $550(0.98)$ & $520(0.98)$ & $520(0.98)$ & $502(0.98)$ & $535(0.97)$ & $516(0.99)$ \\
\hline
\end{tabular}

$F_{(2,34)}=4.78, \mathrm{MSE}=595, p=0.015$. For ipsilateral cues, there was a significant validity $\times$ SOA interaction, $F_{(1,17)}=5.08$, $\mathrm{MSE}=1112, p=0.038$, that did not depend on the stimulation site, $F<1$, or experiment, $F_{(1,17)}=1.93$, MSE $=1112, p=0.182$. For contralateral cues, the validity effect depended on the stimulation site, $F_{(2,34)}=7.04, \mathrm{MSE}=735, p=0.003$, and this was similarly observed in both experiments (interaction between experiment, region and validity, $F<1$ ). Interestingly, however, the lack of short SOA facilitatory effects for contralateral targets under sham TMS persisted, ruling out the responsibility of the right lateralized TMS sound in the absence of short SOA contralateral facilitation.

We finally tested the possibility that at this short SOA, contralateral facilitation might have been very sensitive to practice effects, wearing off in magnitude with training. Such effects would however be unavoidable given the requirement (to ensure a bias-free comparison between sham and active TMS) to respect an accurate counterbalancing across TMS conditions. We thus analyzed the cuing effects of those participants that performed the exogenous sham condition first in the original experiment $(n=2)$, in the control experiment with bilateral sham $(n=2)$, and in two new participants who received sham TMS with a single coil situated on the right (as done in the original experiment). The results showed a significant validity $\times$ SOA interaction, $F_{(1,3)}$ $=89.75, \mathrm{MSE}=69, p=0.002$, that did not depend on cue side (left or right), $F<1$. Facilitatory effects were statistically similar for left and right cues (27 and $31 \mathrm{~ms}$, respectively, $F<1$ ). We therefore conclude that in agreement with previous reports (Weaver et al., 1998; Lupiáñez et al., 2001), the specific absence of short SOA facilitation under sham TMS for contralateral cues is likely to be caused by a practice-related decreases in magnitude for such effect.

\section{Discussion}

Existing fMRI-based network models of attentional orienting in humans have set up a comprehensive framework for discussion. They put forward the hypothesis of a dorsal frontoparietal network in the orienting of both endogenous and exogenous attention, and a ventral frontoparietal counterpart in reorienting to task-relevant events (Corbetta et al., 2008). Nonetheless, the insufficient temporal resolution of such techniques prevents the capture of fast and brief cerebral events, such as exogenously driven attentional orienting, which peaks $\sim 100 \mathrm{~ms}$ after cue onset (Müller and Rabbitt, 1989). Furthermore, the power of fMRI to establish direct causal relations between brain areas and cognitive events is limited and prone to the confounding effects of epiphenomenal brain activations. In this study, we benefited from TMS high temporal and spatial resolution, and its ability to establish causality, to further explore the functional contribution of two parietal regions in the processing of endogenous and exogenous cues. We provide novel information about the role of
IPS on both types of attentional orienting and substantiate a causal link between TPJ and exogenous orienting, which contributes to the refinement of existing depictions for such networks.

Previous TMS studies exploring the lasting — offline-effects of repetitive TMS over temporoparietal sites (Pascual-Leone et al., 1994; Hilgetag et al., 2001; Thut et al., 2005; Meister et al., 2006) have addressed the causal implication of IPS and TPJ in the orienting of attention. However, given the lack of time and event specificity provided by such offline TMS approaches, its impact on other task-associated processes (such as target detection, feature discrimination, or response selection) cannot be ruled out. More specific event-related TMS studies (delivering pulses around the time of target onset) have been applied to dissect out the underlying bases of visual detection or discrimination processes (Rushworth et al., 2001; Grosbras and Paus, 2002; Chambers et al., 2004), but they focused in target onset periods rather than on those specifically underlying cue-induced attentional orienting computations.

Previous studies proposed that endogenous orienting is processed by a bilateral frontoparietal network (Corbetta et al., 1993, 2000; Nobre et al., 1997; Hopfinger et al., 2000) segregated from ventral systems. Accordingly, we specifically attempted to dissociate the ventral and dorsal parietal areas during the cortical processing of exogenous and endogenous cues. Substantiating previous interpretations based on fMRI data, we provide for the first time direct causal evidence of IPS involvement in endogenous and exogenous attentional orienting. These results are compatible with observations suggesting that combined TPJ and parietal damage induce more severe extinction syndromes than the former alone (Friedrich et al., 1998; He et al., 2007). Interestingly, the existence of regions playing a role in both subtypes of spatial orienting suggested by our results, opens an exciting area of future explorations directed at finding out if, as hypothesized previously, some brain regions allow the interaction of both attentional orienting systems to coordinate the final orienting response (Hopfinger and West, 2006; Chica and Lupiáñez, 2009).

At first sight, the paradoxical facilitation driven by IPS stimulation cannot be easily reconciled with models of interhemispheric rivalry (Sprague, 1966; Kinsbourne, 1977; Hilgetag et al., 1999), which have been often invoked to explain other events of parietal TMS driven improvements in visual performance (Pascual-Leone et al., 1994; Hilgetag et al., 2001; Thut et al., 2005). If this were the case, the double pulse interference performed in this study should have punctually released inhibitory connections projecting to left parietal regions, provoking an increase of excitability which should in turn, have biased attentional orienting to ipsilateral rather than contralateral stimuli. A more plausible explanation thus could be that IPS stimulation might have acted by extending the duration of endogenous early facilitatory effects, masking or even interrupting IOR, which ac- 
cording to event-related recordings operates in the system, even if masked in behavioral measures (Wascher and Tipper, 2004; Chica and Lupiáñez, 2009).

The interference of IPS stimulation on the exogenous task found in our study fits well with the hypothesized role of the posterior parietal cortex, in concert with the superior colliculus (SC) (Dorris et al., 2002), as part of the circuitry underlying IOR. The SC is a brainstem structure receiving retinal input, implicated in saccade execution and heavily connected to the FEF (Wurtz and Albano, 1980) and the posterior parietal cortex (Clower et al., 2001). However, collicular direct recordings in the monkey suggest that the SC is not the direct site of inhibition, but rather a structure receiving feedback signals which have already been attenuated in cortical structures such as the parietal cortex (Dorris et al., 2002). Thus TMS on the right IPS may have interfered with the generation of a modulatory signal to the SC, and in consequence suppressed IOR for contralateral stimuli. IPS interference could also have acted locally on the update of saliency maps known to play a role in preventing the remapping of inhibitory tags in such type of paradigms (Sapir et al., 2004; van Koningsbruggen et al., 2010). In any case, magnetic field spread- or connectivity-mediated cross talk between IPS and TPJ could be ruled out on the basis of the estimated spatial selectivity for TMS in parietal cortical areas and of the site-specific effects found in our own study for IPS TMS during the endogenous task.

In addition to its hypothesized contribution to attentional reorienting, our results confirm the role of the right TPJ in the orienting of exogenous spatial attention and show for the first time its causal implication (as previously shown for IPS) in IOR. Different operations as those postulated for IPS might however underlie such a role. TPJ may be specifically important for the detection of novel and behaviorally relevant stimuli (Asplund et al., 2010). A fundamental mechanism contributing to IOR is the relative lack of novelty of a target appearing at the same location of a previously presented cue, separated by long SOA (Milliken et al., 2000). IOR could be caused by a "habituation" of the orienting response some time after the cue has been presented (Milliken et al., 2000; Dukewich, 2009). Such a phenomenon would bias attention toward locations that have not been previously attended or explored. Thus, the absence of inhibition after TPJ transient disruption found in this study could be interpreted as TMS-induced abnormal processing of novelty causing attention to be perseveringly oriented to the cued location (Linden et al., 1999; Downar et al., 2000, 2002; Asplund et al., 2010).

In disagreement with the findings of previous TMS studies, we could not find significant signs of the TMS-driven ipsilateral enhancements (Pascual-Leone et al., 1994; Hilgetag et al., 2001; Thut et al., 2005), for either endogenous or exogenous tasks in our experiments. Nonetheless, notable design and methodological differences such as the absence of a pre-target cue orienting attention, the use of detection instead of discrimination paradigms (Pascual-Leone et al., 1994; Hilgetag et al., 2001), the implementation of crossmodal cuing based on auditory top down signals (Thut et al., 2005), the use of off-line strategies instead of trial by trial "on-line" interference paradigms, or differences in stimulated area and the targeting strategy (Pascual-Leone et al., 1994; Hilgetag et al., 2001; Thut et al., 2005), could account for such discrepancies.

In conclusion, our results provide novel evidence for a causal role for TPJ in the right hemisphere during exogenously but not endogenously driven orienting of attention. Furthermore, we show that right IPS plays a role in both types of orienting. In particular, the manipulation of this area induces a bias in orient- ing at long SOAs resulting in an interference of the IOR phenomenon. Both results contribute to refining and extending existing models of attention orienting circuitry in the human brain. Further work will explore the exact chronometry of such effects, which has only been indirectly addressed in the current project and will extend the current observation to other relevant cortical regions such as the FEF or the left hemisphere IPS and TPJ, previously identified as potentially important in neuroimaging studies.

\section{References}

Asplund CL, Todd JJ, Snyder AP, Marois R (2010) A central role for the lateral prefrontal cortex in goal-directed and stimulus-driven attention. Nat Neurosci 13:507-512.

Bartolomeo P, Chokron S (2002) Orienting of attention in left unilateral neglect. Neurosci Biobehav Rev 26:217-234.

Berlucchi G (2006) Inhibition of return: a phenomenon in search of a mechanism and a better name. Cogn Neuropsychol 23:1065-1074.

Chambers CD, Payne JM, Stokes MG, Mattingley JB (2004) Fast and slow parietal pathways mediate spatial attention. Nat Neurosci 7:217-218.

Chica AB, Lupiáñez J (2009) Effects of endogenous and exogenous attention on visual processing: an inhibition of return study. Brain Res 1278:75-85.

Chica AB, Lupiáñez J, Bartolomeo P (2006) Dissociating inhibition of return from the endogenous orienting of spatial attention: evidence from detection and discrimination tasks. Cogn Neuropsychol 23:1015-1034.

Clower DM, West RA, Lynch JC, Strick PL (2001) The inferior parietal lobule is the target of output from the superior colliculus, hippocampus, and cerebellum. J Neurosci 21:6283-6291.

Corbetta M, Shulman GL (2002) Control of goal-directed and stimulusdriven attention in the brain. Nat Rev Neurosci 3:201-215.

Corbetta M, Miezin FM, Shulman GL, Petersen SE (1993) A PET study of visuospatial attention. J Neurosci 13:1202-1226.

Corbetta M, Kincade JM, Ollinger JM, McAvoy MP, Shulman GL (2000) Voluntary orienting is dissociated from target detection in human posterior parietal cortex. Nat Neurosci 3:292-297.

Corbetta M, Patel G, Shulman GL (2008) The reorienting system of the human brain: from environment to theory of mind. Neuron 58:306-324.

Dorris MC, Klein RM, Everling S, Munoz DP (2002) Contribution of the primate superior colliculus to inhibition of return. J Cogn Neurosci 14:1256-1263.

Downar J, Crawley AP, Mikulis DJ, Davis KD (2000) A multimodal cortical network for the detection of changes in the sensory environment. Nat Neurosci 3:277-283.

Downar J, Crawley AP, Mikulis DJ, Davis KD (2002) A cortical network sensitive to stimulus salience in a neutral behavioral context across multiple sensory modalities. J Neurophysiol 87:615-620.

Dukewich KR (2009) Reconceptualizing inhibition of return as habituation of the orienting response. Psychon Bull Rev 16:238-251.

Friedrich FJ, Egly R, Rafal RD, Beck D (1998) Spatial attention deficits in humans: A comparison of superior parietal and temporal-parietal junction lesions. Neuropsychology 12:193-207.

Grosbras MH, Paus T (2002) Transcranial magnetic stimulation of the human frontal eye field: effects on visual perception and attention. J Cogn Neurosci 14:1109-1120.

Hahn B, Ross TJ, Stein EA (2006) Neuroanatomical dissociation between bottom-up and top-down processes of visuospatial selective attention. Neuroimage 32:842-853.

He BJ, Snyder AZ, Vincent JL, Epstein A, Shulman GL, Corbetta M (2007) Breakdown of functional connectivity in frontoparietal networks underlies behavioral deficits in spatial neglect. Neuron 53:905-918.

Hilgetag CC, Kötter R, Young MP (1999) Inter-hemispheric competition of sub-cortical structures is a crucial mechanism in paradoxical lesion effects and spatial neglect. Prog Brain Res 121:121-141.

Hilgetag CC, Théoret H, Pascual-Leone A (2001) Enhanced visual spatial attention ipsilateral to rTMS-induced 'virtual lesions' of human parietal cortex. Nat Neurosci 4:953-957.

Hopfinger JB, West VM (2006) Interactions between endogenous and exogenous attention on cortical visual processing. Neuroimage 31:774-789.

Hopfinger JB, Jha AP, Hopf JM, Girelli M, Mangun GR (2000) Electrophysiological and neuroimaging studies of voluntary and reflexive attention. 
In: Attention and performance XVIII: the control over cognitive processes (Driver J, Monsell S, eds), pp 125-153. Oxford: Oxford UP.

Kincade JM, Abrams RA, Astafiev SV, Shulman GL, Corbetta M (2005) An event-related functional magnetic resonance imaging study of voluntary and stimulus-driven orienting of attention. J Neurosci 25:4593-4604.

Kinsbourne M (1977) Hemi-neglect and hemisphere rivalry. In: Hemiinattention and hemisphere specialization (Weinstein EA, Friedland RP, eds), pp 41-49. New York: Raven.

Klein RM (2000) Inhibition of return. Trends Cogn Sci 4:138-147.

Klein RM (2004) On the control of visual orienting. In: Cognitive neuroscience of attention (Posner MI, ed), pp 29-44. New York: Guilford.

Linden DE, Prvulovic D, Formisano E, Völlinger M, Zanella FE, Goebel R, Dierks T (1999) The functional neuroanatomy of target detection: an fMRI study of visual and auditory oddball tasks. Cereb Cortex 9:815-823.

Lupiáñez J, Weaver B, Tipper SP, Madrid E (2001) The effects of practice on cueing in detection and discrimination tasks. Psicológica 22:1-23.

Meister IG, Wienemann M, Buelte D, Grünewald C, Sparing R, Dambeck N, Boroojerdi B (2006) Hemiextinction induced by transcranial magnetic stimulation over the right temporo-parietal junction. Neuroscience 142:119-123.

Milliken B, Tipper SP, Houghton G, Lupiáñez J (2000) Attending, ignoring, and repetition: on the relation between negative priming and inhibition of return. Percept Psychophys 62:1280-1296.

Müller HJ, Rabbitt PM (1989) Reflexive and voluntary orienting of visual attention: time course of activation and resistance to interruption. J Exp Psychol Hum Percept Perform 15:315-330.

Nobre AC, Sebestyen GN, Gitelman DR, Mesulam MM, Frackowiak RS, Frith CD (1997) Functional localization of the system for visuospatial attention using positron emission tomography. Brain 120:515-533.

Pascual-Leone A, Gomez-Tortosa E, Grafman J, Alway D, Nichelli P, Hallett
M (1994) Induction of visual extinction by rapid-rate transcranial magnetic stimulation of parietal lobe. Neurology 44:494-498.

Posner MI (1980) Orienting of attention. Q J Exp Psychol 32:3-25.

Posner MI, Cohen Y (1984) Components of visual orienting. In: Attention and performance X (Bouma H, Bouwhuis D, eds), pp 531-556. London: Erlbaum.

Prime DJ, Visser TA, Ward LM (2006) Reorienting attention and inhibition of return. Percept Psychophys 68:1310-1323.

Rushworth MF, Krams M, Passingham RE (2001) The attentional role of the left parietal cortex: the distinct lateralization and localization of motor attention in the human brain. J Cogn Neurosci 13:698-710.

Sapir A, Hayes A, Henik A, Danziger S, Rafal R (2004) Parietal lobe lesions disrupt saccadic remapping of inhibitory location tagging. J Cogn Neurosci 16:503-509.

Sprague JM (1966) Interaction of cortex and superior colliculus in mediation of visually guided behavior in the cat. Science 153:1544-1547.

Thut G, Nietzel A, Pascual-Leone A (2005) Dorsal posterior parietal rTMS affects voluntary orienting of visuospatial attention. Cereb Cortex 15:628-638.

Townsend JT, Ashby FG (1983) Stochastic modeling of elementary psychological processes. New York: Cambridge UP.

van Koningsbruggen MG, Gabay S, Sapir A, Henik A, Rafal RD (2010) Hemispheric asymmetry in the remapping and maintenance of visual saliency maps: a TMS study. J Cogn Neurosci 22:1730-1738.

Wascher E, Tipper SP (2004) Revealing effects of noninformative spatial cues: an EEG study of inhibition of return. Psychophysiology 41:716-728.

Weaver B, Lupiáñez J, Watson FL (1998) The effects of practice on objectbased, location-based, and static-display inhibition of return. Percept Psychophys 60:993-1003.

Wurtz RH, Albano JE (1980) Visual-motor function of the primate superior colliculus. Annu Rev Neurosci 3:189-226. 